\title{
NEGATIVELY CURVED MANIFOLDS WITH EXOTIC SMOOTH STRUCTURES
}

\author{
F. T. FARRELL AND L. E. JONES
}

\section{INTRODUCTION}

Let $M$ denote a compact real hyperbolic manifold with dimension $m \geq 5$ and with sectional curvature $K=-1$. Let $\Sigma_{1}, \Sigma_{2}, \ldots, \Sigma_{k}$ be a complete list of inequivalent exotic spheres of dimension $m$, where two exotic spheres are equivalent provided they are diffeomorphic, but not necessarily via an orientation-preserving diffeomorphism; cf. [14]. (The standard sphere is not included in this list. Note that $k+1$ is the cardinality of the set of equivalence classes of elements $\Sigma$ in the abelian group $\Theta_{m}$ of homotopy spheres, where two elements of $\Theta_{m}$ are equivalent if they are either identical or negatives of each other.) The main result of this paper is the following theorem.

Theorem 1.1. Given any real number $\delta>0$, there is a finite sheeted covering space $\widehat{M}$ of $M$ which satisfies the following properties.

(a) No two of the manifolds $\widehat{M}, \widehat{M} \# \Sigma_{1}, \widehat{M} \# \Sigma_{2}, \ldots, \widehat{M} \# \Sigma_{k}$ are diffeomorphic, but they are all homeomorphic to one another. (Here "\#" denotes connected sum.)

(b) Each of the manifolds $\widehat{M} \# \Sigma_{1}, \widehat{M} \# \Sigma_{2}, \ldots, \widehat{M} \# \Sigma_{k}$ supports a Riemannian metric, all of whose sectional curvature values lie in the interval $(-1-\delta,-1+\delta)$.

Remark. Let $M_{1}$ and $M_{2}$ denote two compact closed connected Riemannian manifolds which have strictly negative sectional curvature values and whose fundamental groups are isomorphic. Since both $M_{1}$ and $M_{2}$ are $K(\pi, 1)$-spaces, it follows that they must be homotopy equivalent to one another. Eells and Sampson showed there is a harmonic map representing a given homotopy class of homotopy equivalences; $\mathrm{Al}^{\prime}$ ber and Hartmann showed this harmonic map is unique; cf. [24, 23, 25]. A problem with some history behind it is to determine whether or not $M_{1}$ and $M_{2}$ must be homeomorphic or diffeomorphic to one another. Cheeger showed in the mid-1970s that the total spaces of the twoframe bundles $V_{2}\left(M_{1}\right), V_{2}\left(M_{2}\right)$ are homeomorphic; cf. [10, 8.2.P]. Gromov then showed that the total spaces of the sphere bundles $S\left(M_{1}\right), S\left(M_{2}\right)$ are

Received by the editors February 10, 1989 and, in revised form, May 29, 1989. This is a revision of a previously (informally) distributed paper. We thank Pat Eberlein and Wolfgang Ziller for very helpful comments on that version, which led to the present more readable one.

1980 Mathematics Subject Classification (1985 Revision). Primary 53C20, 57R55.

Both authors were supported in part by the National Science Foundation. 
homeomorphic via a homeomorphism that preserves the orbits of the geodesic flows; cf. [10, 8.3.E]. Farrell and Hsiang showed in 1979 that $M_{1} \times R^{3}$ and $M_{2} \times R^{3}$ are homeomorphic; cf. [4]. Recently, the authors have shown in [6] that $M_{1}$ and $M_{2}$ are homeomorphic (provided $\operatorname{dim}\left(M_{1}\right) \geq 5$ ); in fact, we showed that any homotopy equivalence between $M_{1}$ and $M_{2}$ is homotopic to a homeomorphism. Whether $M_{1}$ must be diffeomorphic to $M_{2}$ is Problem 12 of the list of geometric problems compiled by Yau [22]. (It is called the Lawson-Yau conjecture.) Theorem 1.1 gives a negative answer to this problem. In fact, $M_{1}$ can have constant sectional curvature equal to -1 while the sectional curvature values of $M_{2}$ can be pinched arbitrarily close to -1 and $M_{1}$ is homeomorphic but not diffeomorphic to $M_{2}$. We note that Gromov showed [9] that too strong a form of pinching would force $M_{2}$ to be diffeomorphic to $M_{1}$. Of course the grandfather of all of these results is Mostow's Rigidity Theorem for hyperbolic space forms [16], which gives a positive answer to Problem 12 when both $M_{1}$ and $M_{2}$ have constant sectional curvature and more generally when both $M_{1}$ and $M_{2}$ are locally symmetric spaces. See Siu [20] and Hamenstadt [12] for generalizations of Mostow's Rigidity Theorem relevant to Problem 12. Also see Mostow and Siu [18] and Gromov and Thurston [11] for other (earlier) examples of negatively curved manifolds which are not diffeomorphic to a locally symmetric space. Theorem 1.1 was motivated by the authors' earlier construction of Anosov diffeomorphisms and expanding endomorphisms on exotic smooth tori $[5,8]$. The situation of positive and zero (both constant and pinched) sectional curvature has been studied by various authors, and it is interesting to compare their results with the negative curvature case. (See [19] for references to these results.)

The proof of Theorem 1.1 depends on the following two propositions.

Proposition 1.2. Suppose that the finite sheeted covering space $\widehat{M}$ of $M$ is stably parallelizable. Then no two of the manifolds $\widehat{M}, \widehat{M} \# \Sigma_{1}, \widehat{M} \# \Sigma_{2}, \ldots, \widehat{M} \# \Sigma_{k}$ are diffeomorphic.

Proposition 1.3. Given a real number $\delta>0$, there is a real number $\alpha>0$ which depends only on $m=\operatorname{dim} M$ and $\delta$ such that the following is true. Suppose that the finite sheeted covering space $\widehat{M}$ of $M$ has radius of injectivity greater than $3 \alpha$ at some point $p \in \widehat{M}$. Then each of the manifolds $\widehat{M} \# \Sigma_{1}, \widehat{M} \# \Sigma_{2}, \ldots, \widehat{M} \# \Sigma_{k}$ supports a Riemannian metric, all of whose sectional curvature values lie in the interval $(-1-\delta,-1+\delta)$.

The proofs of Propositions 1.2 and 1.3 are given in $\S \S 2$ and 3 of this paper, respectively.

We shall now use Propositions 1.2 and 1.3 to complete the proof of Theorem 1.1 .

Proof of Theorem 1.1. First, we use the result of Sullivan [21, p. 553] that there is a finite covering space $M^{\prime}$ of $M$ such that $M^{\prime}$ is stably parallelizable. Let $g_{1}, g_{2}, \ldots, g_{x}$ be a list of all the closed geodesics in $M^{\prime}$ which have length less than or equal to $6 \alpha$, where $\alpha$ comes from Proposition 1.3. Choose a point $q \in M^{\prime}$ and elements $\beta_{1}, \beta_{2}, \ldots, \beta_{x}$ in $\pi_{1}\left(M^{\prime}, q\right)$ such that each 
$\beta_{i}$ is represented by a map $f_{i}: S^{1} \rightarrow M^{\prime}$ which is freely homotopic to $g_{i}$. Since $\pi_{1}\left(M^{\prime}, q\right)$ is residually finite (cf. [16, p. 309]), there is a homomorphism $h: \pi_{1}\left(M^{\prime}, q\right) \rightarrow G$ onto a finite group $G$ such that $h\left(\beta_{i}\right) \neq 1$ for all indices $i$. Let $\widehat{M}$ denote the finite covering space of $M^{\prime}$ corresponding to the kernel of $h$, which is a subgroup of $\pi_{1}\left(M^{\prime}, q\right)$. Note that $\widehat{M}$ is a finite sheeted covering space of $M$ which is stably parallelizable and which has radius of injectivity greater than $3 \alpha$ at each of its points. Thus, we may apply Propositions 1.2 and 1.3 to $\widehat{M}$ to conclude the proof of Theorem 1.1. Note that in proving (a) of Theorem 1.1, we use the general fact that the topological type of a manifold is not changed by forming a connected sum of it with an exotic sphere.

\section{Proof of Proposition 1.2}

We shall need the following lemma to prove Proposition 1.2. Let $N$ be a compact closed topological manifold, and let $N_{1}, N_{2}$ denote two smooth structures on $N$. Recall that $N_{1}$ is said to be concordant to $N_{2}$ if there is a smooth structure $\bar{N}$ on $N \times[0,1]$ such that $\partial_{-} \bar{N}=N_{1} \times 0$ and $\partial_{+} \bar{N}=N_{2} \times 1$. (See [15, p. 24].)

Lemma 2.1. Suppose that the finite sheeted covering space $\widehat{M}$ of $M$ is orientable. Set $N=\widehat{M}, N_{1}=\widehat{M} \# \Sigma_{i}$, and $N_{2}=\widehat{M} \# \Sigma_{j}$. Then $N_{1}$ is concordant to $N_{2}$ if and only if $N_{1}$ is diffeomorphic to $N_{2}$ via an orientation-preserving diffeomorphism.

Proof of Lemma 2.1. First suppose that $N_{1}$ is concordant to $N_{2}$ via a smooth structure $\bar{N}$ for $N \times[0,1]$. Since $\bar{N}$ is topologically a product, it follows from the topological invariance of Whitehead torsion and from the smooth $s$ cobordism theorem that $\bar{N}$ is a product in the smooth category (see [15, pp. 113-116]). Thus, $N_{1}$ is orientation-preservingly diffeomorphic to $N_{2}$, since $N_{1}=\partial_{-} \bar{N}$ and $N_{2}=\partial_{+} \bar{N}$.

On the other hand, if there is a diffeomorphism $f: N_{1} \rightarrow N_{2}$, which preserves orientation, then by Mostow's Rigidity Theorem (see [17]), there is an isometry $g: \widehat{M} \rightarrow \widehat{M}$ such that both $f^{-1}$ and $g$ represent the same element in the outer automorphism group of $\pi_{1} \widehat{M}$. Note that $g$ is isotopic (in the smooth category) to a diffeomorphism $g_{1}: \widehat{M} \rightarrow \widehat{M}$ which is the identity on a ball $B^{m} \subset \widehat{M}$. We may assume that $N_{2}$ is constructed from $\widehat{M}$ by forming the connected sum of $\widehat{M}$ with $\Sigma_{j}$ along the interior of $B^{m}$. Thus, the restriction of $g_{1}$ to $\widehat{M}-B^{m}$ extends to a diffeomorphism $g_{2}: N_{2} \rightarrow N_{2}$. Note that the composite $g_{2} \circ f: N_{1} \rightarrow N_{2}$ is a diffeomorphism which represents the identity element of the outer automorphism group of $\pi_{1} \widehat{M}$. Thus, there is a homotopy $h: \widehat{M} \times[0,1] \rightarrow \widehat{M}$ which restricts to $g_{2} \circ f$ at $\widehat{M} \times 0$ and to the identity map of $\widehat{M}$ at $\widehat{M} \times 1$. Define $H: \widehat{M} \times[0,1] \rightarrow \widehat{M} \times[0,1]$ by $H(x, t)=(h(x, t), t)$. Since $H$ restricts to a homeomorphism on the boundary of $\widehat{M} \times[0,1]$, we may apply [7, Corollary 10.6] to get a homotopy (which is constant on both $\widehat{M} \times 0$ 
and $\widehat{M} \times 1)$ of $H$ to a homeomorphism $H^{\prime}: \widehat{M} \times[0,1] \rightarrow \widehat{M} \times[0,1]$. Note that $H^{\prime}$ satisfies the following properties.

2.2. (a) $H^{\prime}: \widehat{M} \times[0,1] \rightarrow \widehat{M} \times[0,1]$ is a homeomorphism.

(b) $H^{\prime}: \widehat{M} \times 0 \rightarrow \widehat{M} \times 0$ equals the diffeomorphism $g_{2} \circ f: N_{1} \rightarrow N_{2}$.

(c) $H^{\prime}: \widehat{M} \times 1 \rightarrow \widehat{M} \times 1$ is the identity.

Let $\bar{N}$ be the smooth structure on $\widehat{M} \times[0,1]$ which is obtained by pulling back the smooth structure $N_{2} \times[0,1]$ for $\widehat{M} \times[0,1]$ along the map $H^{\prime}$. It follows from 2.2 that $\bar{N}$ is a concordance from $N_{1}$ to $N_{2}$. This completes the proof of Lemma 2.1 .

Let $N_{3}$ denote $\widehat{M} \#\left(-\Sigma_{j}\right)$, and suppose that $f: N_{1} \rightarrow N_{2}$ is an orientationreversing diffeomorphism; then arguing as above would yield a diffeomorphism $g_{3}: N_{2} \rightarrow N_{3}$ such that the composite $g_{3} \circ f: N_{1} \rightarrow N_{3}$ is homotopic to the identity map of $M$ and eventually yields that $N_{1}$ and $N_{3}$ are concordant. In this way, we obtain the following extension of Lemma 2.1.

Addendum 2.3. Suppose $\widehat{M} \# \Sigma_{i}$ is diffeomorphic to $\widehat{M} \# \Sigma_{j}$; then either $\widehat{M} \# \Sigma_{i}$ is concordant to $\widehat{M} \# \Sigma_{j}$ or to $\widehat{M} \#\left(-\Sigma_{j}\right)$. Also, if $\widehat{M} \# \Sigma_{i}$ is diffeomorphic to $\widehat{M}$, then $\widehat{M} \# \Sigma_{i}$ is concordant to $\widehat{M}$.

Proof of Proposition 1.2. Because of Addendum 2.3, it suffices to prove that, for any pair $\left(\Sigma, \Sigma^{\prime}\right)$ of distinct elements in $\Theta_{m}, \widehat{M} \# \Sigma$ is not concordant to $M \# \Sigma^{\prime}$. The proof of this given below is motivated by Brumfiel's paper [3].

Using the fact that $\widehat{M}$ is stably parallelizable and [15, Theorem 4.1, p. 25 ; Theorem 10.1, p. 194], we see that there is a one-to-one correspondence between concordance classes of smooth structures on $\widehat{M}$ and the homotopy classes of maps from $\widehat{M}$ to TOP $/ 0$ (denoted by $[\widehat{M}$, TOP /0]) with the hyperbolic structure on $\widehat{M}$ corresponding to the class of the constant map. For the same reasons, there is a one-to-one correspondence between $\Theta_{m}$ and $\left[S^{m}\right.$, TOP /0] with $S^{m}$ corresponding to the constant class. Let $\beta_{1}$ and $\beta_{2}$ in $\left[S^{m}\right.$, TOP /0] correspond to $\Sigma$ and $\Sigma^{\prime}$, respectively. We recall that TOP $/ 0$ has an infinite delooping associated to the Whitney sum operation (see [2, p. 215]). In particular, TOP $/ 0=\Omega^{2}(X)$ for some space $X$, from which it follows that $[\widehat{M}$, TOP $/ 0]$ has a natural abelian group structure. If $\beta$ is the class of a map from $S^{m}$ to TOP $/ 0$, then $\beta^{*}$ denotes the class of its composite with a degree-one map from $\widehat{M}$ to $S^{m}$. One can compute, based on the naturality of $[15$, Theorem 10.1 , p. 195], that the smooth structures $\widehat{M} \# \Sigma$ and $\widehat{M} \# \Sigma^{\prime}$ correspond to the elements $\beta_{1}^{*}$ and $\beta_{2}^{*}$, respectively. Thus, to complete the proof of Proposition 1.2 , it suffices to verify the following claim.

Claim 2.4. The map $\left[S^{m}, \mathrm{TOP} / 0\right] \stackrel{*}{\rightarrow}[\widehat{M}$, TOP $/ 0]$ (given by sending $\beta$ to $\beta^{*}$ ) is monic, i.e., $\beta_{1}^{*} \neq \beta_{2}^{*}$.

Towards verifying Claim 2.4 , we let $h: \Sigma^{m+2}(\widehat{M}) \rightarrow S^{2 m+2}$ denote the $(m+2)$-fold suspension of a degree-one map $f: \widehat{M} \rightarrow S^{m}$. Since $\widehat{M}$ is stably parallelizable, we have that $\Sigma^{m+2}(\widehat{M})$ is obtained by collapsing to a point 
an $(m+2)$-sphere in the stable Thom-space of $\widehat{M}$. Thus, there is a map $g: S^{2 m+2} \rightarrow \Sigma^{m+2}(\widehat{M})$ realizing the $(m+2)$-fold suspension of the orientation class $[\widehat{M}] \in H_{m}(\widehat{M}, Z)$. Note that the composite $h \circ g: S^{2 m+2} \rightarrow S^{2 m+2}$ has degree equal to one and is therefore homotopic to the identity map. Let $Y$ denote the $(m+2)$-fold delooping of TOP $/ 0$, i.e., $\Omega^{m+2}(Y)=$ TOP $/ 0$ (see [2, p. 215]). Let $\Sigma^{m+2}:[U$, TOP $/ 0] \rightarrow\left[\Sigma^{m+2}(U), Y\right]$ denote the standard suspension isomorphism, and let $f^{*}:\left[S^{m}\right.$, TOP $\left./ 0\right] \rightarrow[\widehat{M}$, TOP $/ 0]$, $g^{*}:\left[\Sigma^{m+2}(\widehat{M}), Y\right] \rightarrow\left[S^{2 m+2}, Y\right]$, and $h^{*}:\left[S^{2 m+2}, Y\right] \rightarrow\left[\Sigma^{m+2}(\widehat{M}), Y\right]$ denote the homomorphisms induced by $f, g$, and $h$. Note that the following properties hold.

2.5. (a) $g^{*} \circ h^{*}=$ identity (because $h \circ g$ is homotopic to the identity).

(b) $\beta_{i}^{*}=f^{*}\left(\beta_{i}\right)$.

(c) $\Sigma^{m+2}$ is an isomorphism.

(d) $\Sigma^{m+2} \circ f^{*}=h^{*} \circ \Sigma^{m+2}$.

Suppose that Claim 2.4 is false, i.e., $\beta_{1}^{*}=\beta_{2}^{*}$. Then using $2.5(\mathrm{a}),(\mathrm{b})$, and (d), we would get that $\Sigma^{m+2}\left(\beta_{1}\right)=\sum^{m+2}\left(\beta_{2}\right)$. Thus, by $2.5(\mathrm{c})$, we have $\beta_{1}=\beta_{2}$, which is a contradiction. This completes the proof of Proposition 1.2.

\section{Proof of Proposition 1.3}

Before beginning the proof of Proposition 1.3, we must recall a fact about manifolds of constant negative curvature and state a lemma.

Let $A($,$) denote the Riemannian metric on S^{m-1} \times(0,3)$ which is the product of the standard Riemannian metric on the unit $(m-1)$-sphere $S^{m-1}$ with the standard Riemannian metric on $(0,3)$. Let $\xi, \gamma$ denote the distributions on $S^{m-1} \times(0,3)$ which are tangent to the first and second factors, respectively. Let $p_{1}: T\left(S^{m-1} \times(0,3)\right) \rightarrow \xi, p_{2}: T\left(S^{m-1} \times(0,3)\right) \rightarrow \gamma$ be the $A$-orthogonal projections. Define 2-tensors $A_{i}(),, i=1,2$, on $S^{m-1} \times(0,3)$ by

$$
A_{i}(v, w)=A\left(p_{i}(v), p_{i}(w)\right)
$$

Define a Riemannian metric $\bar{A}($,$) on S^{m-1} \times(0,3)$ by

3.1. $\bar{A}(v, w)=\sinh ^{2}(\alpha t) A_{1}(v, w)+\alpha^{2} A_{2}(v, w)$ for any pair of vectors $v, w$ tangent to $S^{m-1} \times(0,3)$ at a point $(q, t) \in S^{m-1} \times(0,3)$.

It is well known that if $\widehat{M}$ has radius of injectivity greater than $3 \alpha$ at $p \in \widehat{M}$, there is a smooth map $h: S^{m-1} \times(0,3) \rightarrow \widehat{M}$ which satisfies the following properties.

3.2. (a) $h$ is an embedding.

(b) For each $q \in S^{m-1}$, the path $g(t)=h(q, t)$ is a geodesic of speed $\alpha$ with limit $\operatorname{lin}_{t \rightarrow 0} g(t)=p$.

(c) The pull back along $h: S^{m-1} \times(0,3) \rightarrow \widehat{M}$ of the Riemannian metric $\langle,\rangle_{\widehat{M}}$ is equal to $\bar{A}($,$) .$ 
Remark. To verify 3.2 , we argue as follows. Let $S^{m-1}$ denote the unit sphere centered at the origin in $T \widehat{M}_{p}$, and define $h: S^{m-1} \times(0,3) \rightarrow \widehat{M}$ of 3.2 to be the composite map

$$
S^{m-1} \times(0,3) \stackrel{g}{\rightarrow} T \widehat{M}_{p} \stackrel{\exp }{\rightarrow} \widehat{M},
$$

where $g(v, t)=\alpha t v$. Then $h$ clearly satisfies $3.2(\mathrm{a})$ and (b). To verify $3.2(\mathrm{c})$, it is convenient to choose a coordinate system for $\widehat{M}$ in a disc of radius $3 \alpha$ about $p$ with respect to which $\langle,\rangle_{\widehat{M}}$ has the same coordinates as does the metric in the Poincare disc model for hyperbolic $m$-space. The remaining details are left to the reader.

Note that since $\langle,\rangle_{\widehat{M}}$ has constant sectional curvature equal to -1 , it follows by $3.2(\mathrm{c})$ that $\bar{A}$ also has constant sectional curvature equal to -1 . Another way to see this is by noting that $\bar{A}$ (defined by 3.1 ) is just a special case of the warped product metric of Bishop and O'Neill [1]. Thus, the sectional curvatures of $\bar{A}$ can be computed as in [1, p. 27] to be -1 .

In the following lemma, we consider the sectional curvature for Riemannian metrics on $S^{m-1} \times[1,2]$ which have roughly the same form as $\bar{A} \mid S^{m-1} \times[1,2]$. In more detail, we let $B($,$) be any Riemannian metric on S^{m-1} \times[1,2]$ which satisfies the following properties.

3.3. (a) For any $v \in \xi\left|\left(S^{m-1} \times[1,2]\right), w \in \gamma\right|\left(S^{m-1} \times[1,2]\right)$, we have that $B(v, w)=0$.

(b) If $t$ denotes the second coordinate variable in the product $S^{m-1} \times[1,2]$, then we have that $B(\partial / \partial t, \partial / \partial t)=1$.

We define a new Riemannian metric $\bar{B}\left(\right.$, ) on $S^{m-1} \times[1,2]$ as follows.

3.4. $\bar{B}(v, w)=\sinh ^{2}(\alpha t) B_{1}(v, w)+\alpha^{2} B_{2}(v, w)$ for any pair of vectors $v, w$ tangent to $S^{m-1} \times[1,2]$ at the point $(q, t) \in S^{m-1} \times[1,2]$, where

$$
B_{i}(v, w)=B\left(p_{i}(v), p_{i}(w)\right) .
$$

In the following lemma, we let $P$ denote a 2-plane tangent to $S^{m-1} \times[1,2]$, and we let $K_{\bar{B}}(P)$ denote the sectional curvature of $P$ with respect to $\bar{B}($,$) .$

Lemma 3.5. For a given $B($,$) as in 3.3$ and for $\bar{B}($,$) as in 3.4$, we have

$$
\operatorname{limit}_{\alpha \rightarrow \infty} K_{\bar{B}}(P)=-1
$$

uniformly in $P$.

We will first complete the proof of Proposition 1.3 based on Lemma 3.5. Then we will prove Lemma 3.5.

Proof of Proposition 1.3. Let $E_{+}^{m}, E_{-}^{m}$ denote the northern and southern hemispheres of the unit $m$-sphere $S^{m}$. Each exotic sphere $\Sigma_{i}$ can be constructed by glueing $E_{+}^{m}$ to $E_{-}^{m}$ along a diffeomorphism $f_{i}: \partial E_{+}^{m} \rightarrow \partial E_{-}^{m}$ (this is a well-known application of the $h$-cobordism theorem). Since $\partial E_{ \pm}^{m}=S^{m-1}$, it follows from 3.2 that $\widehat{M} \# \Sigma_{i}$ can be constructed by glueing $S^{m-1} \times[1,2]$ to 
$\widehat{M}-h\left(S^{m-1} \times(1,2)\right)$ along the maps $h: S^{m-1} \times 1 \rightarrow \widehat{M}-h\left(S^{m-1} \times(1,2)\right)$ and $h \circ \bar{f}_{i}: S^{m-1} \times 2 \rightarrow \widehat{M}-h\left(S^{m-1} \times(1,2)\right)$, where $\bar{f}_{i}(x, 2)=\left(f_{i}(x), 2\right)$.

Choose a metric $B($,$) on S^{m-1} \times[1,2]$ which satisfies 3.3 and the following properties.

3.6. Let $B_{1}($,$) be constructed from B($,$) as in 3.4$.

(a) $B_{1}\left|S^{m-1} \times 1=A_{1}\right| S^{m-1} \times 1$.

(b) $B_{1} \mid S^{m-1} \times 2$ equals the pull back along $\bar{f}_{i}: S^{m-1} \times 2 \rightarrow S^{m-1} \times 2$ of $A_{1} \mid S^{m-1} \times 2$.

(c) $B_{1}$ is constant in $t$ near $t=1,2$.

We define a metric $\langle,\rangle_{i}$ on $\widehat{M} \# \Sigma_{i}$ as follows.

$$
\text { (a) }\langle,\rangle_{i}\left|\widehat{M}-h\left(S^{m-1} \times(1,2)\right)=\langle,\rangle_{\widehat{M}}\right| \widehat{M}-h\left(S^{m-1} \times(1,2)\right) \text {. }
$$

(b) $\langle,\rangle_{i} \mid S^{m-1} \times[1,2]=\bar{B}$, where $\bar{B}$ is constructed from $B$ as in 3.4.

It follows from 3.2, 3.6, and 3.7 that $\langle,\rangle_{i}$ is well defined. It follows from 3.7 and from Lemma 3.5 that

$$
\operatorname{limit}_{\alpha \rightarrow \infty} K_{\langle,\rangle_{i}}(P)=-1
$$

uniformly in $P$, where $P$ is any 2-sphere tangent to $M \# \Sigma_{i}$ and where $K_{\langle,\rangle_{i}}(P)$ is the sectional curvature of $P$ with respect to $\langle,\rangle_{i}$.

This completes the proof of Proposition 1.3.

Proof of Lemma 3.5. The proof of the lemma relies upon the following claim.

Claim 3.8. For any $\left(p_{0}, t_{0}\right) \in S^{m-1} \times[1,2]$, there are two coordinate systems $\left(\bar{x}_{1}, \bar{x}_{2}, \ldots, \bar{x}_{m-1}\right)$ and $\left(\bar{y}_{1}, \bar{y}_{2}, \ldots, \bar{y}_{m-1}\right)$ for $S^{m-1}$ near $p_{0}$, and a linear coordinate $\bar{t}$ for $[1,2]$ near $t_{0}$ such that the following hold true.

(a) $\bar{A}(, \quad)=\bar{g}_{i j}^{a} d \bar{x}_{i} d \bar{x}_{j}+d \bar{t}^{2}, \bar{B}(, \quad)=\bar{g}_{i j}^{b} d \bar{y}_{i} d \bar{y}_{j}+d \bar{t}^{2}$, where $\bar{g}_{i j}^{a}=$ $\bar{A}\left(\partial / \partial \bar{x}_{i}, \partial / \partial \bar{x}_{j}\right)$ and $\bar{g}_{i j}^{b}=\bar{B}\left(\partial / \partial \bar{y}_{i}, \partial / \partial \bar{y}_{j}\right)$.

(b) $\bar{g}_{i j}^{a}\left(p_{0}, t_{0}\right)=\delta_{j}^{i}=\bar{g}_{i j}^{b}\left(p_{0}, t_{0}\right)$.

(c) Let $k$ and $s$ denote any nonnegative integers satisfying $k+s \leq 2$, and let $\bar{X}_{i, j ; i_{1} \cdots i_{k}, s}$ and $\bar{Y}_{i, j ; i_{1} \cdots i_{k}, s}$ denote the partial derivatives (through second order)

$$
\frac{\partial^{k+s} \bar{g}_{i j}^{a}}{\partial \bar{x}_{i_{1}} \cdots \partial \bar{x}_{i_{k}} \partial \bar{t}^{s}}\left(p_{0}, t_{0}\right) \text { and } \frac{\partial^{k+s} \bar{g}_{i j}^{b}}{\partial \bar{y}_{i_{1}} \cdots \partial \bar{y}_{i_{k}} \partial \bar{t}^{s}}\left(p_{0}, t_{0}\right)
$$

Then we must have

$$
\operatorname{limit}_{\alpha \rightarrow \infty} \bar{X}_{i, j ; i_{1} \cdots i_{k}, s}=0^{k} 2^{s} \delta_{j}^{i} \text { and } \operatorname{limit}_{\alpha \rightarrow \infty} \bar{Y}_{i, j ; i_{1} \cdots i_{k}, s}=0^{k} 2^{s} \delta_{j}^{i}
$$

uniformly in $\left(p_{0}, t_{0}\right)$ (where $0^{0}=1$ and $0^{k}=0$ if $k \geq 1$ ).

We shall first complete the proof of Lemma 3.5 based on Claim 3.8. Then we shall verify Claim 3.8 .

Choose an orthonormal basis $\left\{v_{1}, v_{2}\right\}$ for the 2-plane $P$, and write $v_{i}=$ $a_{i j} \partial / \partial \bar{y}_{j}+a_{i m} \partial / \partial \bar{t}$, where we sum over the $j$. Set $v_{i}^{\prime}=a_{i j} \partial / \partial \bar{x}_{j}+a_{i m} \partial / \partial \bar{t}$, 
and let $P^{\prime}$ denote the 2-plane spanned by the $\left\{v_{1}^{\prime}, v_{2}^{\prime}\right\}$. Note that it follows from Claim 3.8(a) and (b) and from the classical relation between the coefficients of the curvature tensor and of the first fundamental form (cf. [13, $\S \S 5.3$ and 6.2]), that $K_{\bar{A}}\left(P^{\prime}\right)$ is a polynomial in the $\left\{\bar{X}_{i, j ; i_{1}, \ldots, i_{k}, s}, a_{i j}\right\}$ and that $K_{\bar{B}}(P)$ is the same polynomial in the $\left\{\bar{Y}_{i, j ; i_{1}, \ldots, i_{k}, s}, a_{i j}\right\}$. Thus, by Claim 3.8(c), we have that

$$
\operatorname{limit}_{\alpha \rightarrow \infty}\left(K_{\bar{B}}(P)-K_{\bar{A}}\left(P^{\prime}\right)\right)=0
$$

uniformly in $P$. Since $K_{\bar{A}}\left(P^{\prime}\right)=-1$ (cf. 3.2(c)), the conclusion of Lemma 3.5 follows.

It remains to construct the coordinates $\left(\bar{x}_{1}, \ldots, \bar{x}_{m-1}\right),\left(\bar{y}_{1}, \ldots, \bar{y}_{m-1}\right)$, and $\bar{t}$ and to verify Claim 3.8. Towards this end, we choose coordinates $\left(x_{1}, \ldots, x_{m-1}\right)$ and $\left(y_{1}, \ldots, y_{m-1}\right)$ for $S^{m-1}$ near $p_{0}$ so that the following hold.

3.9. (a) $A()=,g_{i j}^{a} d x_{i} d x_{j}+d t^{2}, B()=,g_{i j}^{b} d y_{i} d y_{j}+d t^{2}$.

(b) $g_{i j}^{a}\left(p_{0}, t_{0}\right)=\delta_{j}^{i}=g_{i j}^{b}\left(p_{0}, t_{0}\right)$.

(c) There is a number $C>0$, which is independent of $\left(p_{0}, t_{0}\right)$, such that for all integers $k, s \geq 0$ with $k+s \leq 2$, the following must hold:

$$
\left|\frac{\partial^{k+s} g_{i j}^{a}}{\partial x_{i_{1}} \cdots \partial x_{i_{k}} \partial t^{s}}\left(p_{0}, t_{0}\right)\right|<C \text { and }\left|\frac{\partial^{k+s} g_{i j}^{b}}{\partial y_{i_{1}} \cdots \partial y_{i_{k}} \partial t^{s}}\left(p_{0}, t_{0}\right)\right|<C \text {. }
$$

Remark. Note that in 3.9(c) all of the smooth functions $\left\{g_{i j}^{a}, g_{i j}^{b}\right\}$ depend on the choice of $\left(p_{0}, t_{0}\right)$. An argument based on the compactness of $S^{m-1} \times[1,2]$ will show that $C$ of 3.9 (c) can be chosen to be independent of $\left(p_{0}, t_{0}\right)$ provided the $\left(x_{1}, \ldots, x_{m-1}\right)$ are chosen to be the normal coordinates for $S^{m-1}$ at $p_{0}$ with respect to the metric $A_{1} \mid S^{m-1} \times t_{0}$ and provided that the $\left(y_{1}, \ldots, y_{m-1}\right)$ are chosen to be the normal coordinates for $S^{m-1}$ at $p_{0}$ with respect to the metric $B_{1} \mid S^{m-1} \times t_{0}$.

Now define the coordinates $\bar{x}_{i}, \bar{y}_{i}$, and $\bar{t}$ as follows:

3.10. (a) $\bar{t}=\alpha t, \bar{t}_{0}=\alpha t_{0} ; \bar{x}_{i}=\sinh \left(\bar{t}_{0}\right) x_{i} ; \bar{y}_{i}=\sinh \left(\bar{t}_{0}\right) y_{i}$.

Then we also have the following equalities.

(b)

$$
d \bar{t}=\alpha d t ; \quad d \bar{x}_{i}=\sinh \left(\bar{t}_{0}\right) d x_{i} ; \quad d \bar{y}_{i}=\sinh \left(\bar{t}_{0}\right) d y_{i}
$$

$$
\frac{\partial}{\partial \bar{t}}=\frac{1}{\alpha} \frac{\partial}{\partial t} ; \quad \frac{\partial}{\partial \bar{x}_{i}}=\frac{1}{\sinh \left(\bar{t}_{0}\right)} \frac{\partial}{\partial x_{i}} ; \quad \frac{\partial}{\partial \bar{y}_{i}}=\frac{1}{\sinh \left(\bar{t}_{0}\right)} \frac{\partial}{\partial y_{i}} .
$$

It follows from 3.9(a), (b) and from 3.10(a), (b) that 3.8(a), (b) are satisfied and that the $\bar{g}_{i j}^{a}, \bar{g}_{i j}^{b}$ of $3.8(\mathrm{a})$ can be computed in terms of the $g_{i j}^{a}, g_{i j}^{b}$ of 3.9(a) as follows:

3.11 .

$$
\bar{g}_{i j}^{a}=\left(\frac{\sinh (\bar{t})}{\sinh \left(\bar{t}_{0}\right)}\right)^{2} g_{i j}^{a} ; \quad \bar{g}_{i j}^{b}=\left(\frac{\sinh \left(\bar{t}_{)}\right.}{\sinh \left(\bar{t}_{0}\right)}\right)^{2} g_{i j}^{b} .
$$



fied.

Finally, note that it follows from $3.9(\mathrm{c}), 3.10(\mathrm{c})$ and 3.11 that $3.8(\mathrm{c})$ is satisThis completes the proof of Lemma 3.5.

\section{REFERENCES}

1. R. L. Bishop and B. O'Neill, Manifolds of negative curvature, Trans. Amer. Math. Soc. $\mathbf{1 4 5}$ (1969), 1-49.

2. J. M. Boardman and R. M. Vogt, Homotopy invariant algebraic structures on topological spaces, Lecture Notes in Math., no. 347, Springer-Verlag, Berlin and New York, 1973.

3. G. Brumfiel, Homotopy equivalence of almost smooth manifolds, Comment. Math. Helv. 46 (1971), 381-407.

4. F. T. Farrell and W.-C. Hsiang, On Novikov's Conjecture for non-positively curved manifolds. I, Ann. of Math. (2) 113 (1981), 199-209.

5. F. T. Farrell and L. E. Jones, Anosov diffeomorphisms constructed from $\pi_{1} \operatorname{Diff}\left(S^{n}\right)$, Topology 17 (1978), 273-282.

6. _ Compact negatively curved manifolds (of $\operatorname{dim} \neq 3,4$ ) are topologically rigid, Proc. Nat. Acad. Sci. U.S.A. 86 (1989), 3461-3463.

7. _ A topological analogue of Mostow's Rigidity Theorem, J. Amer. Math. Soc. 2 (1989), 257-370.

8. __ Examples of expanding endomorphisms on exotic tori, Invent. Math. 45 (1978), 175-179.

9. M. Gromov, Manifolds of negative curvature, J. Differential Geom. 13 (1978), 223-230.

10. _ Hyperbolic groups, Essays in Group Theory (S. M. Gersten, ed.), Math. Sci. Res. Inst. Publ. 8 (1987), 75-263.

11. M. Gromov and W. Thurston, Pinching constants for hyperbolic manifolds, Invent. Math. 89 (1987), 1-12.

12. U. Hamenstadt, A geometric characterization of negatively curved locally symmetric spaces (to appear).

13. N. Hicks, Notes on differential geometry, van Nostrand, Princeton, NJ, 1965.

14. M. Kervaire and J. Milnor, Groups of homotopy spheres: I, Ann. of Math. (2) 77 (1963), 504-537.

15. R. C. Kirby and L. C. Siebenmann, Foundational essays on topological manifolds, smoothings, and triangulations, Ann of Math. Stud., no. 88, Princeton Univ. Press, Princeton, NJ, 1977.

16. W. Magnus, Residually finite groups, Bull. Amer. Math. Soc. 75 (1969), 305-316.

17. G. D. Mostow, Quasi-conformal mappings in $n$-space and the rigidity of hyperbolic space forms, Inst. Hautes Études Sci. Publ. 34 (1967), 53-104.

18. G. D. Mostow and Y. T. Siu, A compact Kähler surface of negative curvature not covered by the ball, Ann. of Math. 112 (1980), 321-360.

19. E. A. Ruh, Almost symmetric spaces, Global Riemannian Geometry, Horwood, Chichester, England, 1984, pp. 93-98.

20. Y. T. Siu, The complex-analyticity of harmonic maps and the strong rigidity of compact Kähler manifolds, Ann. of Math. (2) 112 (1980), 73-111.

21. D. Sullivan, Hyperbolic geometry and homeomorphisms, Geometric Topology (J. Cantrell, ed.), Academic Press, New York, 1979, pp. 543-555.

22. S.-T. Yau, Seminar on differential geometry, Ann. of Math. Stud., no. 102, Princeton Univ. Press, Princeton, NJ, 1982.

23. S. I. Al'ber, Spaces of mappings into manifold of negative curvature, Dokl. Akad. Nauk. SSSR 178 (1968), 13-16. 
24. J. Eells and J. H. Sampson, Harmonic mappings of Riemannian manifolds, Amer. J. Math. 86 (1964), 109-160.

25. P. Hartman, On homotopic harmonic maps, Canad. J. Math. 19 (1967), 673-687.

ABSTRACT. Let $M$ denote a compact real hyperbolic manifold with dimension $m \geq 5$ and sectional curvature $K=-1$, and let $\Sigma$ be an exotic sphere of dimension $m$. Given any small number $\delta>0$, we show that there is a finite covering space $\widehat{M}$ of $M$ satisfying the following properties: the connected sum $\widehat{M} \# \Sigma$ is not diffeomorphic to $\widehat{M}$, but it is homeomorphic to $\widehat{M} ; \widehat{M} \# \Sigma$ supports a Riemannian metric having all of its sectional curvature values in the interval $[-1-\delta,-1+\delta]$. Thus, there are compact Riemannian manifolds of strictly negative sectional curvature which are not diffeomorphic but whose fundamental groups are isomorphic. This answers Problem 12 of the list compiled by Yau [22]; i.e., it gives counterexamples to the Lawson-Yau conjecture. Note that Mostow's Rigidity Theorem [17] implies that $\widehat{M} \# \Sigma$ does not support a Riemannian metric whose sectional curvature is identically -1 . (In fact, it is not diffeomorphic to any locally symmetric space.) Thus, the manifold $\widehat{M} \# \Sigma$ supports a Riemannian metric with sectional curvature arbitrarily close to -1 , but it does not support a Riemannian metric whose sectional curvature is identically -1 . More complicated examples of manifolds satisfying the properties of the previous sentence were first constructed by Gromov and Thurston [11].

Department of Mathematics, Columbia University, New York, New York 10027

Department of Mathematics, SUNy at Stony Brook, Stony Brook, New York 11794 